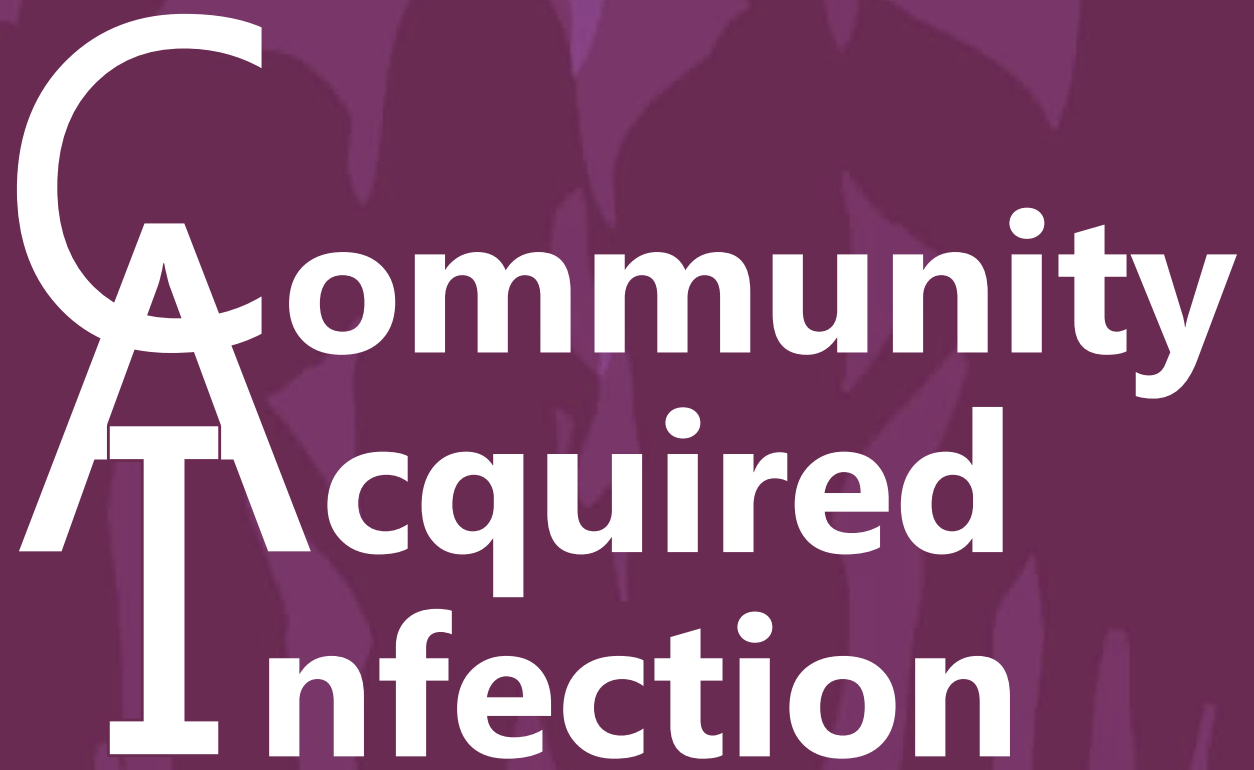

Q. Wolters Kluwer

Medknow
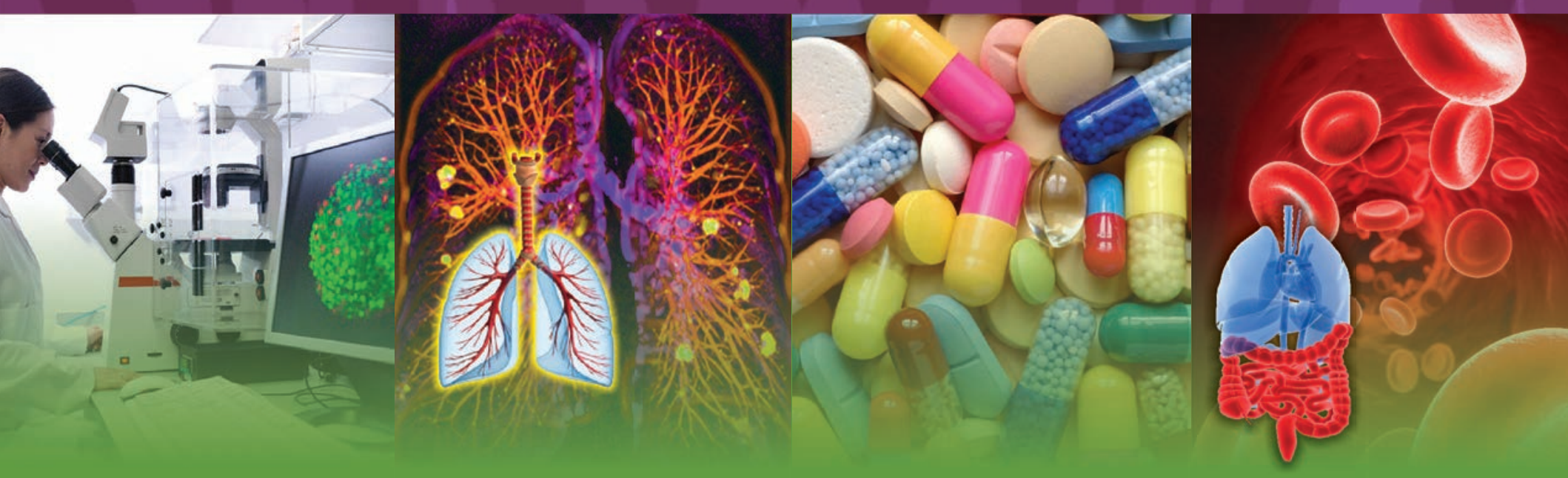

Spring Media Publishing 


\title{
Phenotyping in bronchiectasis: Are we moving toward a personalized medicine?
}

\author{
Paola Faverio, Stefano Carlo Zucchetti, Edoardo Simonetta, Francesco Amati ${ }^{1}$, Andrea Gramegna ${ }^{1}$, \\ Giovanni Sotgiu ${ }^{2}$, Francesco Blasi ${ }^{1}$, Anthony De Soyza ${ }^{3}$, Stefano Aliberti ${ }^{1}$ \\ Department of Cardio-Thoracic-Vascular, University of Milan Bicocca, Respiratory Unit, ASST Monza, San Gerardo Hospital, Monza, \\ ${ }^{1}$ Department of Pathophysiology and Transplantation, University of Milan, Cardio-Thoracic Unit and Cystic Fibrosis Adult Center, \\ Fondazione IRCCS Cà Granda Ospedale Maggiore Policlinico, Milan, ${ }^{2}$ Department of Biomedical Sciences, Clinical Epidemiology and \\ Medical Statistics Unit, University of Sassari, Sassari, Italy, ${ }^{3}$ Institute of Cellular Medicine, Newcastle University, and UK Bronchiectasis \\ Service, Freeman Hospital, Newcastle Upon Tyne, UK
}

\begin{abstract}
Bronchiectasis (BE) is a heterogeneous disease. Similarly, to other chronic airway diseases, such as asthma and chronic obstructive pulmonary disease, management of $\mathrm{BE}$ patients requires a specific and personalized treatment that depends on many clinical, functional, and microbiological variables. Therefore, developing phenotyping methods that can help clinical and therapeutic choices is of paramount importance. Various methodological approaches have been used to personalize patients' management. In this review, we explore the main tools identified so far to classify and phenotype BE patients, including the approaches based on BE etiologies, disease severity, cluster analysis, and endotyping. We also discuss the strengths and limitations of every approach and highlight the similarities and differences between these studies. Finally, we review the therapeutic implications and clinical management connected with each approach.
\end{abstract}

Key words: Bronchiectasis, etiology, severity, inhaled antibiotics, phenotyping

\section{INTRODUCTION}

Bronchiectasis (BE) is a chronic disease of the airways characterized by irreversible dilation of the bronchi. These alterations lead to the impairment of mucociliary clearance and the onset of a vicious circle with persistent bacterial

Address for correspondence:

Prof. Stefano Aliberti, Department of Pathophysiology and Transplantation, Cystic Fibrosis Adult Center, University of Milan, Cardio-thoracic Unit, Fondazione IRCCS Cà Granda Ospedale Maggiore Policlinico, Via Francesco Sforza 35, Milan 20122, Italy. E-mail: stefano.aliberti@unimi.it

\begin{tabular}{|c|l|}
\hline Access this article online & \\
\hline Quick Response Code: & \multirow{2}{*}{ Website: } \\
\hline & www.caijournal.com \\
\cline { 2 - 3 } & \\
\hline & DOI: \\
\hline
\end{tabular}

colonization, chronic inflammation, and progressive tissue destruction. ${ }^{[1]}$ Despite the paucity of data regarding BE prevalence worldwide, a recent population-based study showed that BE is far from being defined a rare disease. ${ }^{[2]}$

BE represents the outcome of a wide group of etiological factors, with patients sharing different clinical manifestations, responses to treatment, and clinical outcomes. ${ }^{[3]}$ In daily clinical practice, the spectrum of the disease can move from mild disease with limited clinical and radiological

\footnotetext{
This is an open access article distributed under the terms of the Creative Commons Attribution-NonCommercial-ShareAlike 3.0 License, which allows others to remix, tweak, and build upon the work non-commercially, as long as the author is credited and the new creations are licensed under the identical terms.

For reprints contact: reprints@medknow.com

How to cite this article: Faverio P, Zucchetti SC, Simonetta E, Amati F, Gramegna A, Sotgiu G, et al. Phenotyping in bronchiectasis: Are we moving toward a personalized medicine?. Community Acquir Infect 2016;3:96-103.
} 
impairment to more severe disease with disseminated pulmonary involvement, severe functional impairment, chronic airways infection by difficult-to-treat pathogens, and frequent exacerbations.

Between these two extreme scenarios, a wide range of clinical syndromes might exist, implying a tailored management approach. So far, there are no specific indications for tailored treatment for BE patients. ${ }^{[3]}$ Over the past few years, several research groups tried to identify a tool to either stratify or cluster BE patients to detect specific groups that might respond to different treatments. ${ }^{[4-9]}$ In the recent past, studies with a similar aim conducted in other respiratory conditions, including chronic obstructive pulmonary disease (COPD) and asthma, have led to more personalized medicine approaches. ${ }^{[10,11]}$

The aim of this review is to evaluate the current literature using various approaches to classify BE patients, with special attention to their clinical aspects and therapeutic perspectives.

\section{AN ETIOLOGY-BASED APPROACH}

The most common etiologies of BE include previous severe respiratory infections (e.g., bacterial pneumonia or tuberculosis), allergic bronchopulmonary aspergillosis (ABPA), impairment of ciliary clearance (e.g., primary ciliary dyskinesia), immunodeficiencies (e.g., hypogammaglobulinemia), and other airway diseases, such as COPD and severe asthma. ${ }^{[3]}$ However, there are many strengths and limitations related to this approach. The identification of treatable causes of BE may prevent the evolution of the disease, and international guidelines recommend to detect them. However, the etiology of $\mathrm{BE}$ leading to targeted therapy is only identified in a minority of cases. ${ }^{[12]}$ This reflects that idiopathic and postinfectious etiologies generally account for $50-70 \%$ of all cases in many case series. ${ }^{[12]}$ There is no agreement on the minimum bundle of tests to be performed to identify BE etiology, and recommendations of the international guidelines are not met in real life. ${ }^{[13]}$ Several etiologies have been associated with BE, but the real "cause-effect" relationships have been proved only in a few cases. Typical examples are the association between $\mathrm{BE}$ and nontuberculous mycobacteria (NTM) or ABPA where the "chicken-and-the egg" story might occur. Both NTM and ABPA might either predispose to the development of $\mathrm{BE}$ or colonize a BE lung. Furthermore, identification of some etiologies of BE, such as the "postinfective" one, suffers significant recall bias related to the difficulties in collecting information on a low respiratory tract infection episode occurred several years before. Finally, no good evidence has been published so far concerning the causality in the association/overlap between $\mathrm{BE}$ and obstructive diseases such as asthma and COPD. ${ }^{[14]}$
According to a recent meta-analysis on the BE etiology, the idiopathic etiology was found in an average of $44.8 \%$ cases, with a high interstudy variability (range: 5-82\%) depending on geographical and diagnostic differences. ${ }^{[12]}$ Apart from idiopathic BE, the other most common etiologies were postinfective (29.9\%), immunodeficiency (5\%), COPD (3.9\%), and connective tissue disease (3.8\%)..$^{[12]}$

All of the latest international guidelines strongly recommend a comprehensive diagnostic workup, including a thorough medical history and baseline investigations, such as full blood count, major immunoglobulin classes, and serological tests for Aspergillus species. ${ }^{[3,15]}$ Furthermore, cultures of airway secretions are strongly recommended because they can detect pathogens, such as NTM. ${ }^{[15]}$ However, according to the meta-analysis published by Gao et al., the identification of the BE etiologies led to changes in patients' management only in $18 \%$ of cases. ${ }^{[12]}$

In a recent paper, Lonni et al. evaluated the possible correlation between $\mathrm{BE}$ etiologies and disease severity. ${ }^{[16]}$ No significant differences in the etiology of BE were found across different levels of disease severity, with the exception of a higher prevalence of COPD-related BE and a lower prevalence of idiopathic BE in patients with severe disease.

Few studies have evaluated patients' phenotyping according to BE etiology [Table 1]. .7-9]

Buscot et al. performed a retrospective, single-center, university hospital-based study on $311 \mathrm{BE}$ patients in Nice, France. ${ }^{[7]}$ They detected three phenotypes with distinct clinical, functional, and microbiological features: idiopathic, congenital, and COPD-associated BE [Table 1]. Anwar et al. identified two main groups: idiopathic $\mathrm{BE}$ and postinfection BE, with the latter being significantly younger at symptom onset compared to the former group. ${ }^{[8]}$ King et al., who collected data from 195 adult BE patients at a University Hospital in Melbourne, Australia, ${ }^{[9]}$ based their phenotyping on the disease onset. Patients with childhood onset, despite the younger age, showed higher disease severity and higher number of exacerbations.

\section{A SEVERITY-BASED APPROACH}

Another approach aimed to classify patients with BE is based on the degree of disease severity. BE severity, ranging from cases without symptoms or exacerbations to patients requiring lung transplantation, greatly impacts on patients' management. Two recognized severity scores are the BE Severity Index (BSI) and the FACED score [Table 2]. ${ }^{[17,18]}$ They share some items, including age, pulmonary function tests (PFTs) with the forced expiratory volume in the in the $\mathrm{l}^{\text {st }}$ second $(\mathrm{FEVl})$, presence of bacterial colonization, radiological extension, and the 
Table 1: Studies reporting bronchiectasis phenotyping according to bronchiectasis etiology and disease onset Buscot et al. 2016[7]

COPD-associated Predominantly men, associated with tobacco use. Pts are older (median age 73 yrs), have more severe airway obstruction at diagnosis and $P$. aeruginosa is more frequently found in the sputum (65\% of cases). Pts have a higher proportion of pulmonary hypertension (16\% of cases).

Congenital Younger pts at diagnosis (median age $20 \mathrm{yrs),} \mathrm{lower} \mathrm{rate} \mathrm{of} \mathrm{smoking} \mathrm{(13 \% ).} \mathrm{Almost} \mathrm{all} \mathrm{of} \mathrm{them} \mathrm{(93 \% )} \mathrm{have} \mathrm{potential}$ pathogens in their sputum ( $P$. aeruginosa in $57 \%$ of cases, $H$. influenzae in $50 \%$, and $S$. aureus in $36 \%$ ). $40 \%$ underwent lobectomy.

Idiopathic Mostly women, rarely smokers. Pts have better spirometry values at diagnosis and are less likely to carry P. aeruginosa ( $30 \%$ of cases).

Anwar et al. 2013 ${ }^{[8]}$

Idiopathic

Pts in this group are older at symptoms onset (mean age $42 \mathrm{yrs}$ ) and with reduced diagnostic delay compared to "post-infective" etiology (13 yrs).

Post-infective Pts reporting a history of symptoms onset within $10 \mathrm{yrs}$ of a severe respiratory tract infection, such as pneumonia, whooping cough or complicated measles infection. It includes post-tuberculous BE. Pts in this group are younger at symptoms onset (mean age $19 \mathrm{yrs}$ ) but with prolonged diagnostic delays (26 yrs). They are more likely to have a history of childhood infections, including pneumonia. They are also more likely to have a history of rhinitis and lobectomy.

King et al. 2009[9]

Childhood onset

Mostly women with longer duration of productive cough; higher prevalence of rhinosinusitis, exacerbations per year (mean 3/yr) and presence of crepitations on physical exam. Worse PFTs and radiological alterations. Higher proportion of adequate sputum specimens for microbiological analysis and higher incidence of $P$. aeruginosa.

Adult onset Higher age at diagnosis (mean age $61 \mathrm{yrs)} \mathrm{with} \mathrm{shorter} \mathrm{duration} \mathrm{of} \mathrm{symptoms.} \mathrm{Lower} \mathrm{number} \mathrm{of} \mathrm{exacerbations} \mathrm{(mean}$ $2 / y r$ ). Less severe radiological and functional involvement. Lower volume of sputum and higher incidence of no growth on sputum specimens.

BE: Bronchiectasis, pts: Patients, yrs: years, COPD: Chronic obstructive pulmonary disease.

Table 2: Bronchiectasis Severity Scores

\begin{tabular}{|c|c|c|c|c|}
\hline \multirow{2}{*}{ FEV1\% predicted } & \multicolumn{2}{|c|}{ Bronchiectasis Severity Index (17) } & \multicolumn{2}{|c|}{ FACED score (18) } \\
\hline & $>80$ & 0 points & $\geq 50 \%$ & 0 points \\
\hline & $50-80$ & 1 point & $<50 \%$ & 2 points \\
\hline & $30-49$ & 2 points & & \\
\hline & $<30$ & 3 points & & \\
\hline \multirow[t]{4}{*}{ Age } & $<50$ years & 0 points & $<70$ years & 0 points \\
\hline & $50-69$ years & 2 points & $\geq 70$ years & 2 points \\
\hline & $70-79$ years & 4 points & & \\
\hline & $\geq 80$ years & 6 points & & \\
\hline \multirow[t]{3}{*}{ Chronic bacterial colonization } & P. aeruginosa & 3 points & No & 0 points \\
\hline & other organisms & 1 point & Yes, with $P$. aeruginosa & 1 point \\
\hline & None & 0 points & & \\
\hline \multirow[t]{2}{*}{ Number of lobes with BE } & $\leq 2$ & 0 points & $\leq 2$ & 0 points \\
\hline & $>2$ or cystic $\mathrm{BE}$ & 1 point & $>2$ & 1 point \\
\hline \multirow[t]{3}{*}{ Dyspnea, mMRC score } & $\leq 3$ & 0 points & $\leq 2$ & 0 points \\
\hline & 4 & 2 points & $>2$ & 1 point \\
\hline & 5 & 3 points & & \\
\hline \multirow[t]{2}{*}{ Body mass index } & $<18.5$ & 2 points & & \\
\hline & $\geq 18.5$ & 0 points & & \\
\hline \multirow[t]{2}{*}{ Hospital admissions in the past 2 years } & Yes & 5 points & & \\
\hline & No & 0 points & & \\
\hline \multirow[t]{2}{*}{ Exacerbation frequency in the past 12 months } & $<3$ & 0 points & & \\
\hline & $\geq 3$ & 2 points & & \\
\hline
\end{tabular}

BSI: 0-4 points, low risk of hospitalization and mortality, 5-8 points: moderate risk of hospitalization and mortality, $\geqslant 9$ points: high risk of hospitalization and mortality. FACED score: low risk 5-year all-cause mortality (0-2 points), moderate risk of mortality (3-4 points), high risk of mortality (5-7 points). FEV1: Forced expiratory volume in the $1^{\text {st }}$ second, BE: Bronchiectasis, mMRC scale: Modified Medical Research Council Scale, BSI: Bronchiectasis Severity, yrs: years, COPD: Chronic obstructive pulmonary disease.

presence of dyspnea (evaluated through the modified Medical Research Council Scale [mMRC]). These scoring systems were created to predict patients at high risk of mortality, hospitalization, and exacerbations in the case of the BSI, or only mortality in the case of the FACED score. Recently, Li et al. from China created and validated a scoring system for identifying patients at risk of exacerbations. ${ }^{[19]}$ Most of the proposed items were similar to those by Chalmers et al. and Martínez-García et al., including age, dyspnea evaluated through the mMRC scale, Pseudomonas aeruginosa colonization, extension of radiological involvement, and PFTs impairment. The authors also included the presence of prior intensive care unit admission. 
Several studies compared the BSI and the FACED scores. ${ }^{[20-22]}$ Ellis et al. found that both scoring systems had similar predictive power for 5 -year mortality, with the FACED score showing slightly superior predictive power for 15-year mortality. [21] The BSI was also developed to predict the risk of hospitalization and exacerbations. A recently published study by McDonnell et al. directly compared BSI and FACED scores for the assessment of clinically relevant disease outcomes across seven European cohorts. ${ }^{[22]}$ The study showed that both scores accurately predicted mortality, but BSI was superior to FACED in predicting multiple outcomes, including hospital admissions, exacerbations, quality of life, and lung function decline. The ability of predicting not only mortality but also other clinically relevant outcomes supports the utility of BSI in "real-life" decision-making processes. Patients at high risk may benefit from aggressive treatment while those at low-risk could receive nonspecialist follow-up or simpler treatment regimens. Data to validate this approach are now needed.

While severity scores provide an important piece of the puzzle formed by the patient and their disease, they do not allow a personalized approach, with, for instance, two very different clinical cases characterized by similar BSI or FACED scores and different clinical management [Table 3].

\section{PHENOTYPING ACCORDING TO CLINICAL AND MICROBIOLOGICAL CHARACTERISTICS WITH A CLUSTER-BASED APPROACH}

The third approach that has emerged more recently is cluster-based analysis.

Cluster analysis refers to a group of multivariate mathematical algorithms that broadly perform two distinct functions: (1) quantification of similarity between individuals within a population on the basis of (multiple) specified variables; (2) grouping of individuals into clusters such that similarity between members of the same clusters is strong, while similarities between members in different clusters is weak. ${ }^{[11,23]}$ Furthermore, to validate the primary cluster analysis, independent external cohorts are recruited.

In recent years, several phenotypes for asthma and COPD have been proposed. ${ }^{[10,11]}$ Two kinds of phenotypes of asthmatic patients have been used: clinical (e.g., allergic, aspirin-sensitive, and glucocorticoid-resistant asthma ${ }^{[24]}$ ) and pathophysiological phenotypes (e.g., eosinophilic, neutrophilic, mixed inflammatory, and paucigranulocytic asthma). ${ }^{[25]}$ These approaches are sometime referred to (clinical) phenotyping and endotyping, respectively.

The former category is helpful to clinicians because it can be detected through clinical history and physical examination. The latter category is important for pharmacologists, allowing the development of therapies targeted to the pathophysiological mechanisms of the disease. The distinction between phenotypes allows the clinician to identify the best-individualized treatment.

Focusing on BE, to date, there are three main studies describing clinical phenotyping.

Aliberti et al. performed a principal component and cluster analyses using data from five European BE registries (1145 patients). ${ }^{[4]}$ The authors identified four clinical phenotypes mainly based on microbiological isolations and respiratory symptoms [Table 4]. These groups differ from one another both from a therapeutic and prognostic point of view. In the Pseudomonas cluster, more than $50 \%$ of the patients were exposed to a macrolide, and more than one-third were on inhaled antibiotic treatment; this cluster also showed higher rates of exacerbations, hospitalizations, and mortality rate during the 3-year follow-up period. One-third of the patients in the "other chronic infection" and "daily sputum" clusters were on long-term macrolides, while few patients received inhaled antibiotic therapy; these two clusters also shared a similar 3 -year mortality rate and number of hospitalizations at 1-year follow-up. The "dry BE" cluster showed the best outcomes and rarely received antibiotic maintenance therapy.

Guan et al. performed a hierarchical cluster analysis on a data set of 148 adult Chinese patients with BE. ${ }^{[5]}$ Clusters were defined by patient's age at symptoms' onset and past clinical history [Table 4]. Cluster \#2 and, secondly, Cluster \#3, showed similarities with the Pseudomonas group of the study by Aliberti et al. These two clusters showed more extensive radiological and functional involvement, which translated into a greater severity of disease with more respiratory symptoms and worse quality of life. P. aeruginosa colonization was common in both groups. Furthermore, similarly to the Pseudomonas cluster, these two groups showed high rates of macrolide therapy, increased number of BE exacerbations in the previous 2 years and increased health-care utilization. Guan et al. also described cluster \# 1, which showed mild disease severity and shared some characteristics with the "dry BE" group by Aliberti et al. The main feature of this cluster was the reduced health-care utilization.

Martínez-García et al. in a recently published multicenter study described the results of a cluster analysis involving 468 patients from six Spanish BE reference centers. ${ }^{[6]}$ The most severe cluster of patients described in this cohort is the "elderly/severe/exacerbators" phenotype. This group shared many characteristics with the Pseudomonas and "daily sputum" phenotypes by Aliberti et al. and Clusters 2-3 in the study by Guan et al. In fact, patients in this group were the oldest, the most symptomatic not only for breathlessness but also for purulent sputum production, the most likely to have an active 
Table 3: Different clinical scenarios with similar Bronchiectasis Severity Index and FACED score

\begin{tabular}{|c|c|}
\hline \multicolumn{2}{|l|}{ Bronchiectasis Severity Index } \\
\hline CASE 1 & CASE 2 \\
\hline $\begin{array}{l}\text { Older underweight adult with BE-COPD overlap syndrome } \\
\text { and emphysema, no chronic bacterial colonization, } \\
\text { moderate-to-severe dyspnea and functional impairment. No } \\
\text { frequent exacerbator. } \\
\text { - FEV } 40 \% \text { predicted } \rightarrow 2 \text { points } \\
\text { - Age } 75 \text { yrs } \rightarrow 4 \text { points } \\
\text { - No chronic bacterial colonization } \rightarrow 0 \text { points } \\
\text { - } 2 \text { lobes with } B E \rightarrow 0 \text { points } \\
\text { - mMRC score }=4 \rightarrow 2 \text { points } \\
\text { - Body mass index }=18 \rightarrow 2 \text { points } \\
\text { - No hospital admissions in the past } 2 \text { yrs } \rightarrow 0 \text { points } \\
\text { - } 2 \text { exacerbations in the past } 12 \text { months } \rightarrow 0 \text { points }\end{array}$ & $\begin{array}{l}\text { Young adult with idiopathic BE, no functional impairment, chronic } P \text {. } \\
\text { aeruginosa colonization and frequent exacerbator. One hospitalization in the } \\
\text { prior year due to pneumonia. } \\
\text { - FEV } 82 \% \text { predicted } \rightarrow 0 \text { points } \\
\text { - Age } 40 \text { yrs } \rightarrow 0 \text { points } \\
\text { - Chronic } P \text {. aeruginosa colonization } \rightarrow 3 \text { points } \\
\text { - } 2 \text { lobes with } B E \rightarrow 0 \text { points } \\
\text { - mMRC score }=1 \rightarrow 0 \text { points } \\
\text { - Body mass index }=21 \rightarrow 0 \text { points } \\
\text { - } 1 \text { hospital admission in the past } 2 \text { yrs } \rightarrow 5 \text { points } \\
\text { - } 3 \text { exacerbations in the past } 12 \text { months } \rightarrow 2 \text { points }\end{array}$ \\
\hline $\begin{array}{l}\text { Total score } 10 \\
\text { FACED score }\end{array}$ & Total score 10 \\
\hline CASE 3 & CASE 4 \\
\hline $\begin{array}{l}\text { Same as Case } 1 \text {. } \\
\text { - FEV } 140 \% \text { predicted } \rightarrow 2 \text { points } \\
\text { - Age } 75 \text { yrs } \rightarrow 2 \text { points } \\
\text { - No chronic bacterial colonization } \rightarrow 0 \text { points } \\
\text { - } 2 \text { lobes with } B E \rightarrow 0 \text { points } \\
\text { - mMRC score }=4 \rightarrow 1 \text { point }\end{array}$ & $\begin{array}{l}\text { Young adult with post-infective } \mathrm{BE} \text { and immunodeficiency, moderate dyspnea, } \\
\text { radiological and functional impairment. Chronic } P \text {. aeruginosa colonization. } \\
-\mathrm{FEV}_{1} 50 \% \text { predicted } \rightarrow 2 \text { points } \\
\text { - Age } 40 \text { yrs } \rightarrow 0 \text { points } \\
\text { - Chronic } P \text {. aeruginosa colonization } \rightarrow 1 \text { point } \\
\text { - } 3 \text { lobes with } \mathrm{BE} \rightarrow 1 \text { points } \\
\text { - mMRC score }=3 \rightarrow 1 \text { point }\end{array}$ \\
\hline Total score 5 & Total score 5 \\
\hline
\end{tabular}

BE: Bronchiectasis, yrs: years, COPD: Chronic obstructive pulmonary disease, FEV : Forced expiratory volume in the $1^{\text {st }}$ second, mMRC scale: Modified Medical Research Council Scale.

or prior smoking habit and to have COPD as comorbidity. Furthermore, this cluster showed a high prevalence of P. aeruginosa and Haemophilus influenzae colonization. Chronic treatment with macrolides and inhaled antibiotics were used in $34 \%$ and $46 \%$ of cases, respectively. This group showed the worst outcomes, including a high number of exacerbations in the prior year and an increased mortality for respiratory causes. Martínez-García et al. also described a phenotype defined as "elderly/severe/non-exacerbators" that, differently from all the phenotypes described in the other studies, was characterized by a high mortality rate, but mainly due to non-respiratory causes (cardiovascular diseases and neoplasms). Interestingly, these patients showed relatively low chronic colonization with H. influenzae and P. aeruginosa, low number of exacerbations in the prior year, and low use of chronic macrolides and inhaled antibiotics. Probably, the poor outcomes in this cluster were mainly associated with comorbidities and not with BE itself.

The phenotypes presented so far are very useful from a clinical point of view; however, their usefulness in understanding the biological mechanisms and pathophysiology of the disease is less clear and further studies are required.

As observed by the previously described studies, some phenotypes may present similar characteristics. This is the case for patients in the BE-COPD overlap syndrome group by Buscot et al., the Pseudomonas and "daily sputum" phenotypes by Aliberti et al., Cluster \#3 by Guan et al., and the "elderly/severe/exacerbators" phenotype by
Martínez-García et al. ${ }^{[4-7]}$ In particular, BE patients with COPD are usually elderly, very symptomatic subjects with multiple comorbidities, and severe functional impairment.

The differences and similarities between the proposed phenotypes reflect the heterogeneity of patients worldwide and the need to individualize clustering at local and national level.

\section{ENDOTYPING OF BRONCHIECTASIS}

In the era of targeted therapies in asthma, certain endotypes have emerged, including high and low eosinophils and periostin groups. ${ }^{[26,27]}$ The characterization of BE endotypes could include the evaluation of cellular and soluble factors. A recent study by Dente et al. showed higher levels of neutrophilic bronchial inflammation in BE patients with bacterial colonization, especially with $P$. aeruginosa and elevated BSI. ${ }^{[28]}$ Higher levels of free neutrophil elastase activity, myeloperoxidase activity, and interleukin (IL)-l $\beta$ were also found by Aliberti et al. in the "Pseudomonas" and "other chronic infection" clusters, confirming the high levels of neutrophil inflammatory markers in patients with bacterial colonization. ${ }^{[4]}$

Some soluble inflammatory markers and, particularly a deregulated cytokine network, including IL-8, tumor necrosis factor-alpha, and neutrophil elastase are also plausible candidates in BE pathogenesis and, thus, potentially useful in endotyping. ${ }^{[29]}$ 
Table 4: Studies reporting bronchiectasis phenotyping according to clinical and microbiological characteristics with a cluster-based approach

Aliberti et al. 2016 ${ }^{[4]}$

Cluster 1 - "Pseudomonas"

Cluster 2 - "Other chronic infection"

Cluster 3 - "Daily sputum"

Cluster 4 - "Dry bronchiectasis"

Presence of respiratory symptoms (mainly chronic cough, hemoptysis, and dyspnea) and extensive radiological impairment. More severe functional alterations involving PFTs and performance status, with increased need for LTOT and worse quality of life. Increased activation of the inflammatory system during stable state. All these factors lead to a higher severity and to a more rapid progression of the disease, which ultimately translates into an increased number of exacerbations and hospitalizations and a higher 3-year mortality. Pts in this group show more often a post-infectious etiology. Chronic infection with bacteria other than $P$. aeruginosa.

Presence of daily sputum production even in absence of bacterial colonization. These pts are more often smokers or ex-smokers. Pts in this group show more often a COPD-related etiology.

Lower disease severity, with the lowest level of inflammatory biomarkers, the least severe radiological and less functional impairment, as well as reduced frequency of respiratory symptoms (in particular, absence of daily sputum production). None of these pts have chronic respiratory infections.

Guan et al. 2016 ${ }^{[5]}$

Cluster 1

Younger patients (mean age $40 \mathrm{yrs}$ ) with shorter time since symptoms onset, with predominantly mild and idiopathic $\mathrm{BE}$, and with minor healthcare resource use. Pts have minimal functional and radiological alterations and only mildly impaired quality of life. Most patients show no colonization with pathogenic bacteria $(80 \%)$, and low risk of BE exacerbations.

Cluster 2 Young pts (mean age $41 \mathrm{yrs}$ ) with the longest duration of symptoms. Post-infectious etiology predominates. Pts have greater disease severity, with more extensive radiological impairment, poorer lung function, higher rate of airway $P$. aeruginosa colonisation (59\%), and frequent health care resource use. This cluster comprises the highest number of pts with cystic BE (96\%) and atelectasis (55\%). 6-minute walk distance is the lowest among all clusters, accompanied by low quality of life.

Cluster 3 Elderly pts with shorter duration of symptoms and mostly idiopathic BE. This cluster presents the most severe form of $\mathrm{BE}$ both radiologically and functionally. Most pts have $P$. aeruginosa colonization $(63 \%)$. A considerable number of pts have cystic BE $(94 \%)$, emphysema $(44 \%)$, pulmonary cavities $(75 \%)$ and bilateral BE $(100 \%)$. Pts in this group have the lowest quality of life and the greatest risk of experiencing pulmonary exacerbations. The use of healthcare resources is frequent.

Cluster 4 Elderly pts with modest time since symptoms onset and moderate disease severity. A few patients have $P$. aeruginosa colonization (17\%). This group shows modest use of healthcare resources without quality of life impairment.

Martínez-García et al. 2016[6]

Young/mild phenotype

Young women with low BMI, mild disease and no frequent exacerbators. Absence of airflow obstruction. Main BE etiologies are: genetic/immune deficiency, post-infectious or idiopathic. Low rate of non-respiratory comorbidities and chronic bronchial infections.

Elderly/mild phenotype Elderly overweight women with mild disease and no frequent exacerbators. Presence of mild airflow obstruction. Main BE etiologies: idiopathic, post-infectious or asthma. Low rate of non-respiratory comorbidities and chronic bronchial infections.

Elderly/severe/exacerbator Elderly men with moderate-severe disease, high prevalence of chronic bronchial infection, severe flow obstruction, and frequent exacerbations. Main BE etiologies: post-infectious and COPD-associated. Low rate of non-respiratory comorbidities.

Elderly/severe/non-exacerbator phenotype

Elderly pts with moderate-severe disease, severe flow obstruction, low number of exacerbations and moderate chronic bronchial infection rate. Main etiologies: post-infectious and idiopathic. High rate of non-respiratory comorbidities (cardiovascular and neoplasms).

These findings suggest that further multicenter studies on $\mathrm{BE}$ phenotyping-endotyping are required to better understand both the pathogenesis of the disease and possible therapeutic targets.

This approach has already lead to new therapeutic advances in asthma. ${ }^{[30]}$

In BE patients, Wells et al. have recently demonstrated "blocking antibodies" that protect $P$. aeruginosa from complement-mediated killing in a very severe subpopulation with $P$. aeruginosa infection. ${ }^{[31]}$ This abnormality of humoral response appears to be characterized by excess production of O-antigen-specific IgG2 antibodies and patients with this "endotype" have more marked disease severity. This form of endotyping may allow us to tailor-specific treatments beyond that suggested by the clinical phenotyping alone.

\section{THERAPEUTIC IMPLICATIONS AND CLINICAL MANAGEMENT}

BE patients grouping on the basis of clinical, microbiological, and etiological criteria have implications for the prognosis and the therapeutic management of the disease. Patients may require individualized treatments based on clinical phenotypes.

On the one hand, phenotypes such as "dry BE" in the study by Aliberti et al..$^{[4]}$ and patients not colonized by pathogenic bacteria, with few respiratory symptoms and low severity of 
the disease, do not usually require a close follow-up. Those patients after an initial assessment with general therapeutic indications (e.g., airway clearance techniques, prevention of exacerbations/respiratory infections, and bronchodilation if indicated) may be followed up by the general practitioner. Some data suggest that up to $30 \%$ of these patients may acquire $P$. aeruginosa, so ongoing microbiological surveillance, albeit at a low frequency, may be helpful. ${ }^{[32]}$

On the other hand, patients with chronic infections, particularly those with $P$. aeruginosa, require closer follow-up; in those cases, monitoring of sputum cultures, both during exacerbations and stable state, becomes of primary importance. These patients are candidates for targeted therapy, using inhalational antibiotics, for bacterial eradication or reduction of bacterial load.

Recently, a single-center retrospective study on the real-life use of nebulized antibiotics aimed to characterize the clinical phenotype of patients that most benefit from such treatment. ${ }^{[33]} \mathrm{BE}$ patients with a greater number of exacerbations, a lower lung function, a greater presence of nodules and cysts on computed tomography scan, and higher BSI score seemed to represent a sort of "higher risk" cluster of patients that showed the best outcomes after aerosolized antibiotics.

Other particular BE phenotypes may require specific therapeutic approaches. As an example, COPD-associated BE may require counseling for smoking cessation and personalized rehabilitation programs. Such patients may also be the ones, in whom targeted screening for alpha-l antitrypsin deficiency provides a better yield than other etiologies.

\section{FUTURE PROSPECTIVES}

Although much progress has been achieved recently in characterizing BE both from a prognostic and clinical point of view, many advances are still needed in the therapeutic field. A better knowledge of the BE etiology, with specific reference to treatable causes ${ }^{[3]}$ the increasingly widespread use of long-term macrolide therapy, ${ }^{[34-36]}$ as well as a greater attention to the prevention of $\mathrm{BE}$ exacerbations, and to the role of the respiratory therapists have definitely improved clinical practice. However, the issue of airways bacterial colonization, which seems to be one of the most important factors in the severity of the disease, should be adequately addressed, particularly in terms of pulmonary targeted antimicrobial therapy. Both nebulized and dry powder inhaled antibiotics are available for use in cystic fibrosis. ${ }^{[37]}$ To date, the available studies mainly focused on P. aeruginosa chronic infection, reported worse outcomes, with bacterial eradication only in a few cases. ${ }^{[38-40]}$ Furthermore, nebulized antibiotics may be associated with side effects (e.g., five times increase in bronchospasm reported with inhaled aminoglycosides compared to placebo). ${ }^{[41]}$ Therefore, better selection of the candidates for inhaled antibiotics, as previously described, as well as new molecules or better formulations of the available ones could improve patients' outcomes.

Finally, in regard to phenotyping, future studies should aim to assess the applicability in clinical practice of the previously described clusters and their power to predict differential responses to therapy, to inform on the design of future randomized control trials.

\section{CONCLUSIONS}

The need of personalizing the management of $\mathrm{BE}$ patients has been internationally recognized as a research priority in the field. ${ }^{[42]}$ The elements that seem to better define the severity of BE phenotypes are the presence of chronic bronchial infections, primarily with $P$. aeruginosa, secondly, with other bacteria, and the presence of chronic respiratory symptoms. The evaluation of the etiology of BE suffers from several limitations, and the need of collecting data at a multi-country level seems to play a secondary role, with the only exception of COPD-associated BE. ${ }^{[43]}$

\section{Financial support and sponsorship \\ Nil.}

\section{Conflicts of interest}

There are no conflicts of interest.

\section{REFERENCES}

1. Chalmers JD, Aliberti S, Blasi F. Management of bronchiectasis in adults. Eur Respir J 2015;45:1446-62.

2. Ringshausen FC, de Roux A, Diel R, Hohmann D, Welte T, Rademacher J. Bronchiectasis in Germany: A population-based estimation of disease prevalence. Eur Respir J 2015;46:1805-7.

3. Pasteur MC, Bilton D, Hill AT; British Thoracic Society Bronchiectasis Non-CF Guideline Group. British Thoracic Society guideline for non-CF bronchiectasis. Thorax 2010;65 Suppl 1:i1-58.

4. Aliberti S, Lonni S, Dore S, McDonnell MJ, Goeminne PC, Dimakou K, et al. Clinical phenotypes in adult patients with bronchiectasis. Eur Respir J 2016;47:1113-22.

5. Guan WJ, Jiang M, Gao YH, Li HM, Xu G, Zheng JP, et al. Unsupervised learning technique identifies bronchiectasis phenotypes with distinct clinical characteristics. Int J Tuberc Lung Dis 2016;20:402-10.

6. Martínez-García MÁ, Vendrell M, Girón R, Máiz-Carro L, de la Rosa Carrillo D, de Gracia J, et al. The multiple faces of non-cystic fibrosis bronchiectasis. A cluster analysis approach. Ann Am Thorac Soc 2016;13:1468-75.

7. Buscot $\mathrm{M}$, Pottier $\mathrm{H}$, Marquette $\mathrm{CH}$, Leroy S. Phenotyping adults with non-cystic fibrosis bronchiectasis: A 10-year cohort study in a French regional university hospital center. Respiration 2016;92:1-8.

8. Anwar GA, McDonnell MJ, Worthy SA, Bourke SC, Afolabi G, Lordan J, et al. Phenotyping adults with non-cystic fibrosis bronchiectasis: A prospective observational cohort study. Respir Med 2013;107:1001-7.

9. King PT, Holdsworth SR, Farmer M, Freezer N, Villanueva E, Holmes PW. Phenotypes of adult bronchiectasis: Onset of productive cough in childhood and adulthood. COPD 2009;6:130-6. 
10. Han MK, Agusti A, Calverley PM, Celli BR, Criner G, Curtis JL, et al. Chronic obstructive pulmonary disease phenotypes: The future of COPD. Am J Respir Crit Care Med 2010;182:598-604.

11. Haldar P, Pavord ID, Shaw DE, Berry MA, Thomas M, Brightling CE, et al. Cluster analysis and clinical asthma phenotypes. Am J Respir Crit Care Med 2008;178:218-24.

12. Gao YH, Guan WJ, Liu SX, Wang L, Cui JJ, Chen RC, et al. Aetiology of bronchiectasis in adults: A systematic literature review. Respirology 2016;21:1376-83.

13. Aliberti S, Hill AT, Mantero M, Battaglia S, Centanni S, Lo Cicero S, et al. Quality standards for the management of bronchiectasis in Italy: A national audit. Eur Respir J 2016;48:244-8.

14. Hurst JR, Elborn JS, De Soyza A; BRONCH-UK Consortium. COPD-bronchiectasis overlap syndrome. Eur Respir J 2015;45:310-3.

15. Chang AB, Bell SC, Torzillo PJ, King PT, Maguire GP, Byrnes CA, et al. Chronic suppurative lung disease and bronchiectasis in children and adults in Australia and New Zealand Thoracic Society of Australia and New Zealand guidelines. Med J Aust 2015;202:130.

16. Lonni S, Chalmers JD, Goeminne PC, McDonnell MJ, Dimakou K, et al. Etiology of non-cystic fibrosis bronchiectasis in adults and its correlation to disease severity. Ann Am Thorac Soc 2015; 12:1764-70.

17. Chalmers JD, Goeminne P, Aliberti S, McDonnell MJ, Lonni S, Davidson J, et al. The bronchiectasis severity index. An international derivation and validation study. Am J Respir Crit Care Med 2014; 189:576-85.

18. Martínez-García MÁ, de Gracia J, Vendrell Relat M, Girón RM, Máiz Carro L, de la Rosa Carrillo D, et al. Multidimensional approach to non-cystic fibrosis bronchiectasis: The FACED score. Eur Respir J 2014;43:1357-67.

19. Li J, Jiao R, Wen LF, Du XB, Gao Y, Li XY, et al. The derivation and validation of a scoring system for identifying patients with bronchiectasis at risk of exacerbations. Zhonghua Jie $\mathrm{He} \mathrm{He} \mathrm{Hu} \mathrm{Xi}$ Za Zhi 2016;39:598-602.

20. Minov J, Karadzinska-Bislimovska J, Vasilevska K, Stoleski S, Mijakoski D. Assessment of the non-cystic fibrosis bronchiectasis severity: The FACED Score vs. the bronchiectasis severity index. Open Respir Med J 2015;9:46-51.

21. Ellis HC, Cowman S, Fernandes M, Wilson R, Loebinger MR. Predicting mortality in bronchiectasis using bronchiectasis severity index and FACED scores: A 19-year cohort study. Eur Respir J 2016;47:482-9.

22. McDonnell MJ, Aliberti S, Goeminne PC, Dimakou K, Zucchetti SC, Davidson $\mathrm{J}$, et al. Multidimensional severity assessment in bronchiectasis: An analysis of seven European cohorts. Thorax 2016. pii: Thorax jnl-2016-208481.

23. Hartigan JA. Clustering. Annu Rev Biophys Bioeng 1973;2:81-101.

24. Kiley J, Smith R, Noel P. Asthma phenotypes. Curr Opin Pulm Med 2007; 13:19-23.

25. Green $\mathrm{RH}$, Brightling $\mathrm{CE}$, Bradding $\mathrm{P}$. The reclassification of asthma based on subphenotypes. Curr Opin Allergy Clin Immunol 2007; 7:43-50.

26. Stokes JR, Casale TB. Characterization of asthma endotypes: Implications for therapy. Ann Allergy Asthma Immunol 2016;117:121-5

27. Izuhara K, Ohta S, Ono J. Using periostin as a biomarker in the treatment of asthma. Allergy Asthma Immunol Res 2016;8:491-8.

28. Dente FL, Bilotta M, Bartoli ML, Bacci E, Cianchetti S, Latorre M, et al. Neutrophilic bronchial inflammation correlates with clinical and functional findings in patients with noncystic fibrosis bronchiectasis. Mediators Inflamm 2015;2015:642503.

29. Fuschillo S, De Felice A, Balzano G. Mucosal inflammation in idiopathic bronchiectasis: Cellular and molecular mechanisms. Eur Respir J 2008;31:396-406.

30. Perlikos F, Hillas G, Loukides S. Phenotyping and endotyping asthma based on biomarkers. Curr Top Med Chem 2016;16:1582-6.

31. Wells TJ, Whitters D, Sevastsyanovich YR, Heath JN, Pravin J, Goodall $\mathrm{M}$, et al. Increased severity of respiratory infections associated with elevated anti-LPS IgG2 which inhibits serum bactericidal killing. J Exp Med 2014;211:1893-904.

32. McDonnell MJ, Jary HR, Perry A, MacFarlane JG, Hester KL, Small T, et al. Non cystic fibrosis bronchiectasis: A longitudinal retrospective observational cohort study of Pseudomonas persistence and resistance. Respir Med 2015; 109:716-26.

33. Nadig TR, Flume PA. Aerosolized antibiotics for patients with bronchiectasis. Am J Respir Crit Care Med 2016;193:808-10.

34. Serisier DJ, Martin ML, McGuckin MA, Lourie R, Chen AC, Brain $B$, et al. Effect of long-term, low-dose erythromycin on pulmonary exacerbations among patients with non-cystic fibrosis bronchiectasis: The BLESS randomized controlled trial. JAMA 2013;309:1260-7.

35. Wong C, Jayaram L, Karalus N, Eaton T, Tong C, Hockey H, et al. Azithromycin for prevention of exacerbations in non-cystic fibrosis bronchiectasis (EMBRACE): A randomised, double-blind, placebo-controlled trial. Lancet 2012;380:660-7.

36. Altenburg J, de Graaff CS, Stienstra Y, Sloos JH, van Haren EH, Koppers RJ, et al. Effect of azithromycin maintenance treatment on infectious exacerbations among patients with non-cystic fibrosis bronchiectasis: The BAT randomized controlled trial. JAMA 2013;309:1251-9.

37. Quon BS, Goss CH, Ramsey BW. Inhaled antibiotics for lower airway infections. Ann Am Thorac Soc 2014;11:425-34.

38. Tay GT, Reid DW, Bell SC. Inhaled antibiotics in cystic fibrosis (CF) and non-CF bronchiectasis. Semin Respir Crit Care Med 2015;36:267-86.

39. Chalmers JD, Loebinger M, Aliberti S. Challenges in the development of new therapies for bronchiectasis. Expert Opin Pharmacother 2015; 16:833-50.

40. Finch S, McDonnell MJ, Abo-Leyah H, Aliberti S, Chalmers JD. A comprehensive analysis of the impact of pseudomonas aeruginosa colonization on prognosis in adult bronchiectasis. Ann Am Thorac Soc 2015;12:1602-11.

41. Brodt AM, Stovold E, Zhang L. Inhaled antibiotics for stable non-cystic fibrosis bronchiectasis: A systematic review. Eur Respir J 2014;44:382-93.

42. Aliberti S, Masefield S, Polverino E, De Soyza A, Loebinger MR, Menendez $\mathrm{R}$, et al. Research priorities in bronchiectasis: $A$ consensus statement from the EMBARC clinical research collaboration. Eur Respir J 2016;48:632-47.

43. Chalmers JD, Aliberti S, Polverino E, Vendrell M, Crichton M, Loebinger $\mathrm{M}$, et al. The EMBARC European bronchiectasis registry: Protocol for an international observational study. ERJ Open Res 2016;2. pii: 00081-2015. 